\title{
OPTIMIZING RANGE NORM OF THE IMAGE SET OF MATRIX OVER INTERVAL MAX-PLUS ALGEBRA
}

\section{Siswanto $^{1,2}$, Ari Suparwanto ${ }^{2}$ and M. Andy Rudhito ${ }^{3}$}

${ }^{1}$ Mathematics Department

Faculty of Mathematics and Natural Science

Sebelas Maret University

Indonesia

e-mail: sis.mipauns@yahoo.co.id

${ }^{2}$ Mathematics Department

Faculty of Mathematics and Natural Science

Gadjah Mada University

Indonesia

e-mail: ari_suparwanto@yahoo.com

${ }^{3}$ The Study Program in Mathematics Education

Faculty of Teacher Training and Education

Sanata Dharma University

Indonesia

e-mail: arudhito@yahoo.co.id

\begin{abstract}
Let $\mathbb{R}$ be the set of real numbers and $\mathbb{R}_{\varepsilon}=\mathbb{R} \cup\{\varepsilon\}, \quad \varepsilon=-\infty$. Maxplus algebra is the set $\mathbb{R}_{\varepsilon}$ that is equipped with two operations maximum and addition. It can be formed matrices in the size of $m \times n$,
\end{abstract}

Received: June 8, 2015; Revised: August 30, 2015; Accepted: September 16, 2015

2010 Mathematics Subject Classification: 15A80.

Keywords and phrases: interval max-plus algebra, range norm, image set.

Communicated by K. K. Azad 
whose elements belong to $\mathbb{R}_{\varepsilon}$, called matrix over max-plus algebra. Let $I(\mathbb{R})_{\varepsilon}=\{x=[\underline{x}, \bar{x}] \mid \underline{x}, \bar{x} \in \mathbb{R}, \varepsilon<\underline{x} \leq \bar{x}\} \cup\{\varepsilon\}$ and $\varepsilon=\{\varepsilon, \varepsilon\}$. The set $I(\mathbb{R})_{\varepsilon}$ which is equipped with two operations maximum and addition is called interval max-plus algebra. It can be formed matrices in the size $m \times n$, whose elements belong to $I(\mathbb{R})_{\varepsilon}$, called matrices over interval max-plus algebra. Optimizing range norm of the image set of matrix over max-plus algebra has been discussed. In this paper, we discuss optimizing range norm of the image set of matrix over interval max-plus algebra.

\section{Introduction}

Max-plus algebra is the set $\mathbb{R}_{\varepsilon}=\mathbb{R} \bigcup\{\varepsilon\}$, of which $\mathbb{R}$ are all the sets of real numbers and $\varepsilon=-\infty$ that is equipped with two operations $\oplus$ (maximum) and $\otimes$ (addition). Max-plus algebra has been used to model and analyze problems in planning, communication, production system, queueing system with finite capacity, parallel computation, and traffic using algebra [3] and also several other applications, i.e., in flight scheduling and airport problems [2] whereas min-plus algebra is the set $\mathbb{R}_{\varepsilon^{\prime}}=\mathbb{R} \bigcup\left\{\varepsilon^{\prime}\right\}$ and $\varepsilon^{\prime}=\infty$ that is equipped with two operations $\oplus^{\prime}$ (minimum) and $\otimes^{\prime}$ (addition) [12]. Tam [12] also discussed complete max-plus algebra, of which the set $\overline{\mathbb{R}}_{\varepsilon}=$ $\mathbb{R} \cup\left\{\varepsilon, \varepsilon^{\prime}\right\}$ is equipped with operations $\oplus$ and $\otimes$, and complete min-plus algebra, of which the set $\overline{\mathbb{R}}_{\mathcal{\varepsilon}^{\prime}}=\mathbb{R} \cup\left\{\varepsilon, \varepsilon^{\prime}\right\}$ is equipped with two operations $\oplus^{\prime}$ and $\otimes^{\prime}$. Furthermore, $\overline{\mathbb{R}}_{\varepsilon}$ and $\overline{\mathbb{R}}_{\varepsilon^{\prime}}$ are written as $\overline{\mathbb{R}}$.

It can be formed a set of matrices in the size $m \times n$ of the sets $\mathbb{R}_{\varepsilon}, \mathbb{R}_{\mathcal{\varepsilon}^{\prime}}$, and $\overline{\mathbb{R}}$. The set of matrices with components in $\mathbb{R}_{\varepsilon}$ is denoted by $\mathbb{R}_{\varepsilon}^{m \times n}$. The matrix $A \in \mathbb{R}_{\varepsilon}^{m \times n}$ is called matrix over max-plus algebra. For $n=1$, a set of vectors over max-plus algebra is obtained and written as $\mathbb{R}_{\varepsilon}^{m}$, that is $\mathbb{R}_{\varepsilon}^{m}=\left\{\left(x_{1}, x_{2}, \ldots, x_{m}\right)^{T} \mid x_{1}, x_{2}, \ldots, x_{m} \in \mathbb{R}_{\varepsilon}\right\}[5,6]$. Moreover, if $m=n$,

then the set $\mathbb{R}_{\varepsilon}^{n \times n}$ equipped with operations $\oplus$ and $\otimes$ forms idempotent semiring $[1,7,10]$. 
Tam [12] illustrated an example of application of max-plus algebra in the production system. For example $A=\left(A_{i j}\right) \in \mathbb{R}_{\varepsilon}^{n \times n}$ is a production matrix, where $A_{i j}$ shows the period of time of the production process from machine $M_{j}$ to $M_{i}$, while $x(k)=\left(x_{i}(k)\right) \in \mathbb{R}_{\varepsilon}^{n}$ is a vector, where $x_{i}(k)$ is the starting time of machine $M_{i}$ at the $k$ th stage. In this production process, we have the equation $x(k+1)=A \otimes x(k)$. One of the criteria used by manufacturer is that the production process is expected to be continue periodically in certain periods, e.g., $\lambda$, so that the equation $x(k+1)=\lambda \otimes$ $x(k)$ is obtained. From $x(k+1)=A \otimes x(k)$ and $x(k+1)=\lambda \otimes x(k)$, we get $A \otimes x(k)=\lambda \otimes x(k)$. The eigenvalue and eigenvector problem of matrix $A$ is to determine the eigenvalue $\lambda$ and the eigenvector $x(k)$ which satisfy the equation $A \otimes x(k)=\lambda \otimes x(k)$.

In the real life situation, there are several ways for the manufacturer to determine the starting time of each machine. One of the ways is by choosing eigenvector as the starting time, so that the system will immediately reach the steady state; that is the process continues periodically with the eigenvalue as its periods. However, in reality, there can exist more than one independent eigenvectors for the manufacturer to choose. In that case, a set of linear combination of the independent eigenvectors is constructed, and as such, the manufacturer needs an additional criterion to choose one element of the set. The additional criterion is by considering the difference between the largest and the smallest of the starting time of each machine. The difference is expressed as range norm of the starting time of each machine. Manufacturer can optimize (either minimize or maximize) the range norm of the starting time of each machine [4, 12]. The readiness of the raw material, the availability of the resources and the distribution of the product become the determining factors for the manufacturer to optimize the range norm of the starting time of each machine. In solving the problems of that production system, Tam [12] has discussed optimizing range norm of the image set of matrix over max-plus algebra. 
Kreinovich [8] stated that any measurement is never $100 \%$ accurate. Thus, the value of the measurement $\tilde{x}$ in general differs from the real value $x$. Specifically, in the case of interval uncertainty, after it is being measured and the result of the measurement $\tilde{x}$ is obtained, the acquired information that the real value $x$ is contained in the interval $x=[\tilde{x}-\Delta, \tilde{x}+\Delta]$.

Based on the above statement, the estimation on the period of time of a certain process can be given, for example the period of time of the process in a production system. Therefore, the period of time of a certain production process can be given in an interval of time.

According to the Kreinovich's idea [8] about the probability to give an estimation of the period of time of a certain process and Rudhito [9] about max-plus algebra generalization, i.e., interval max-plus algebra, we try to expand the concept in max-plus algebra, i.e., the optimizing range norm of the image set of matrix over interval max-plus algebra. Siswanto et al. [11] have investigated how to minimize range norm of the image set of matrix over interval max-plus algebra. In this research, we develop the range norm definition in [11]. Using the new range norm definition, we investigate the way to minimize and maximize range norm of the image set of matrix over interval max-plus algebra.

Before discussing the result of this paper, several concepts which support the discussion are observed as follows [12]:

Definition 1.1. Given that $a \in \mathbb{R}$, the conjugate of $a$ is $a^{*}=a^{-1}=-a$. Suppose that $A \in \overline{\mathbb{R}}^{m \times n}$. Then the conjugate of matrix $A$ is $A^{*}=\left(a_{j i}^{*}\right)$ or $A^{*}=-A^{T}$.

Definition 1.2. Given that $A \in \overline{\mathbb{R}}^{m \times n}$ and if matrix $A$ at least has one finite element in each row, then matrix $A$ is called row $\mathbb{R}$-astic. If matrix $A$ at least has one finite element in each column, then matrix $A$ is called column $\mathbb{R}$-astic. If matrix $A$ at least has one finite element in each row and each column, then matrix $A$ is called double $\mathbb{R}$-astic. 
Definition 1.3. Suppose that $A \in \mathbb{R}_{\varepsilon}^{m \times n}, \operatorname{Im}(A)$ is defined as $\operatorname{Im}(A)=$ $\left\{A \otimes x \mid x \in \mathbb{R}_{\varepsilon}^{n}\right\}$, namely the image set of matrix $A$.

Moreover, the definition of the range norm and the problem of optimizing range norm of the image set of matrix over max-plus algebra are given. The notations of $M=\{1,2, \ldots, m\}$ and $N=\{1,2, \ldots, n\}$ are used to simplify.

Definition 1.4. If $x \in \mathbb{R}^{m}$, then the function $\delta(x)=\sum_{i \in M}^{\oplus} x_{i}-\sum_{i \in M}^{\oplus^{\prime}} x_{i}$ is called the range norm of $x$. In other words, the range norm of $x=$ the largest value in $x$ - the smallest value in $x$.

In this case, the norm term is a mere identification, in contrast to the norm defined in inner product space in general.

Problem 1.1. Given that matrix $A \in \mathbb{R}_{\varepsilon}^{m \times n}$, solve:

$$
\begin{aligned}
& \text { minimize } \delta(b) \\
& \text { subject to } b \in \operatorname{Im}(A) \text {. }
\end{aligned}
$$

Definition 1.5. If $x \in \mathbb{R}_{\varepsilon}^{m}$, then the function $\tilde{\delta}(x)=\sum_{\substack{i \in M \\ x_{i} \neq \varepsilon}}^{\oplus} x_{i}-\sum_{\substack{i \in M \\ x_{i} \neq \varepsilon}}^{\oplus^{\prime}} x_{i}$ is the range norm of $x$, considering only the finite component of $x$.

Problem 1.2. Given that matrix $A \in \mathbb{R}_{\varepsilon}^{m \times n}$, solve:

$$
\begin{aligned}
& \text { minimize } \tilde{\delta}(b) \\
& \text { subject to } b \in \operatorname{Im}(A) \text {. }
\end{aligned}
$$

Problem 1.3. Given that matrix $A \in \mathbb{R}_{\varepsilon}^{m \times n}$, solve:

maximize $\delta(b)$

subject to $b \in \operatorname{Im}(A)$.

Other than the above concepts, the concepts on interval max-plus algebra is also needed [9]. 
Closed interval $x$ in $\mathbb{R}_{\varepsilon}$ is a subset of $\mathbb{R}_{\varepsilon}$ in the form $x=[\underline{x}, \bar{x}]=$ $\left\{x \in \mathbb{R}_{\varepsilon} \mid \underline{x} \leq x \leq \bar{x}\right\}$. The interval $x$ in $\mathbb{R}_{\varepsilon}$ is known as interval max-plus. A number $x \in \mathbb{R}_{\varepsilon}$ can be expressed as interval $[x, x]$.

Definition 1.6. $I(\mathbb{R})_{\varepsilon}=\{x=[\underline{x}, \bar{x}] \mid \underline{x}, \bar{x} \in \mathbb{R}, \varepsilon<\underline{x} \leq \bar{x}\} \bigcup\{\varepsilon\}$ is formed with $\varepsilon=[\varepsilon, \varepsilon]$. On the set $I(\mathbb{R})_{\varepsilon}$, the operations $\bar{\oplus}$ and $\bar{\otimes}$ with $x \bar{\oplus} y=$ $[\underline{x} \oplus \underline{y}, \bar{x} \oplus \bar{y}]$ and $x \bar{\otimes} y=[\underline{x} \otimes \underline{y}, \bar{x} \otimes \bar{y}]$ for every $x, y \in I(\mathbb{R})_{\varepsilon}$ are defined. Furthermore they are known as interval max-plus algebra and notated as $I(\Re)_{\max }=\left(I(\mathbb{R})_{\varepsilon} ; \bar{\oplus}, \bar{\otimes}\right)$.

Definition 1.7. The set of matrices in the size $m \times n$ with the elements in $I(\mathbb{R})_{\varepsilon}$ is notated as $I(\mathbb{R})_{\varepsilon}^{m \times n}$, namely:

$$
I(\mathbb{R})_{\varepsilon}^{m \times n}=\left\{A=\left[A_{i j}\right] \mid A_{i j} \in I(\mathbb{R})_{\varepsilon} ; \text { for } i=1,2, \ldots, m \text { and } j=1,2, \ldots, n\right\}
$$

Matrices which belong to $I(\mathbb{R})_{\varepsilon}^{m \times n}$ are known as matrices over interval max-plus algebra or shortly interval matrices.

Definition 1.8. For $A \in I(\mathbb{R})_{\varepsilon}^{m \times n}$, matrices $\underline{A}=\left[\underline{A}_{i j}\right] \in \mathbb{R}_{\varepsilon}^{m \times n}$ and $\bar{A}=$ $\left[\bar{A}_{i j}\right] \in \mathbb{R}_{\varepsilon}^{m \times n}$ are defined, each is known as lower bound matrix and upper bound matrix of the interval matrix $A$.

Definition 1.9. Given that interval matrix $A \in I(\mathbb{R})_{\varepsilon}^{m \times n}$, of which $\underline{A}$ and $\bar{A}$ each act as lower bound matrix and upper bound matrix of the interval matrix $A$. The matrix interval of $A$ is defined, namely $[\underline{A}, \bar{A}]=$ $\left\{A \in \mathbb{R}_{\varepsilon}^{m \times n} \mid \underline{A} \leq A \leq \bar{A}\right\}$ and $I\left(\mathbb{R}_{\varepsilon}^{m \times n}\right)_{b}=\left\{[\underline{A}, \bar{A}] \mid A \in I(\mathbb{R})_{\varepsilon}^{m \times n}\right\}$.

The semimodule $I(\mathbb{R})_{\varepsilon}^{n \times n}$ over $I(\mathbb{R})_{\varepsilon}$ is isomorphic to the semimodule $I\left(\mathbb{R}_{\varepsilon}^{n \times n}\right)_{b}$ over $I(\mathbb{R})_{\varepsilon}$ with the mapping of $f: I(\mathbb{R})_{\varepsilon}^{n \times n} \rightarrow I\left(\mathbb{R}_{\varepsilon}^{n \times n}\right)_{b}$, $f(A)=[\underline{A}, \bar{A}], \quad \forall A \in I(\mathbb{R})_{\varepsilon}^{n \times n}$. The matrix interval $[\underline{A}, \bar{A}] \in I\left(\mathbb{R}_{\varepsilon}^{n \times n}\right)_{b}$ is 
known as matrix interval which corresponds to interval matrix $A \in I(\mathbb{R})_{\mathcal{\varepsilon}}^{n \times n}$ and which is represented with $A \approx[\underline{A}, \bar{A}]$.

Definition 1.10. It is defined that

$$
I(\mathbb{R})_{\varepsilon}^{n}=\left\{x=\left[x_{1}, x_{2}, \ldots, x_{n}\right]^{T} \mid x_{i} \in I(\mathbb{R})_{\varepsilon} ; i=1,2, \ldots, n\right\} .
$$

Set $I(\mathbb{R})_{\varepsilon}^{n}$ can be considered as set $I(\mathbb{R})_{\varepsilon}^{n \times 1}$. The elements of $I(\mathbb{R})_{\varepsilon}^{n}$ are called interval vector over $I(\mathbb{R})_{\varepsilon}$. Interval vector $x$ corresponds to vector interval $[\underline{x}, \bar{x}]$, i.e., $x \approx[\underline{x}, \bar{x}]$.

The concept of interval min-plus algebra is defined in the same way with interval max-plus algebra concepts. Following are the definitions of complete interval max-plus algebra and complete interval min-plus algebra [11].

Definition 1.11. Complete interval max-plus algebra is set $I(\overline{\mathbb{R}})_{\varepsilon}=$ $I(\mathbb{R})_{\varepsilon} \cup\left\{\varepsilon^{\prime}\right\}, \quad \varepsilon^{\prime}=\left[\varepsilon^{\prime}, \varepsilon^{\prime}\right]$ that is completed with two operations $\bar{\oplus}$ and $\bar{\otimes}$, meanwhile complete interval min-plus algebra is set $I(\overline{\mathbb{R}})_{\varepsilon^{\prime}}=I(\mathbb{R})_{\mathcal{\varepsilon}^{\prime}} \bigcup\{\varepsilon\}$ with the operations $\bar{\oplus}^{\prime}$ and $\bar{\otimes}^{\prime}$. Furthermore, $I(\overline{\mathbb{R}})_{\varepsilon}=I(\mathbb{R})_{\mathcal{E}^{\prime}}$ are written as $I(\overline{\mathbb{R}})$.

In the same way as in the interval max-plus algebra and interval min-plus algebra, in complete interval max-plus algebra and complete interval minplus algebra, can be defined as the set of matrices in the size $m \times n$ are notated as $I(\overline{\mathbb{R}})^{m \times n}$.

\section{Main Results}

In this section, the optimizing range norm of the image set of matrix over interval max-plus algebra is presented:

\section{A. Minimizing the range norm of matrix image set over interval max- plus algebra}

The following are definitions of the range norm of vector and the image set of matrix over interval max-plus algebra. 
Definition 2.1. Suppose that $x \in I(\mathbb{R})^{m}$ with $x \approx[\underline{x}, \bar{x}], \underline{x}, \bar{x} \in \mathbb{R}^{m}$. The function $I \delta(x)=[\min (\delta(\underline{x}), \delta(\bar{x})), \delta(\bar{x})]$ is called the range norm of $x$. In other words, the range norm of $x$ is interval $[\min (\delta(\underline{x}), \delta(\bar{x}))$, $\delta(\bar{x})]$, where $\min (\delta(\underline{x}), \delta(\bar{x}))=$ minimum of $\delta(\underline{x})$ or $\delta(\bar{x})$.

Definition 2.2. Given that matrix $A \in I(\mathbb{R})_{\varepsilon}^{m \times n}$ with $A \approx[\underline{A}, \bar{A}]$, $\underline{A}, \bar{A} \in \mathbb{R}_{\varepsilon}^{m \times n}$, it is then defined that $\operatorname{Im}(A)=\left\{A \bar{\otimes} p \mid p \in I(\mathbb{R})_{\varepsilon}^{n}\right\}$.

Based on the above definitions, the problem of minimizing the range norm of matrix image set can be formulated as follows:

Problem 2.1. Given that matrix $A \in I(\mathbb{R})_{\mathcal{\varepsilon}}^{m \times n}$, solve:

$$
\begin{aligned}
& \text { minimize } I \delta(b) \\
& \text { subject to } b \in \operatorname{Im}(A) \text {. }
\end{aligned}
$$

Definition 2.3. Given that matrix $b \in I(\mathbb{R})_{\varepsilon}^{m}$ with $b \approx[\underline{b}, \bar{b}]$. The function $I \delta(b)$ is said minimum if and only if $\delta(\underline{b})$ and $\delta(\bar{b})$ are minimum.

If the image vector has infinite components, the definition about the range norm and the problem of minimizing the range norm of matrix image set can be formulated as follows:

Definition 2.4. Suppose that $x \in I(\mathbb{R})_{\varepsilon}^{m}$ with $x \approx[\underline{x}, \bar{x}]$. The function $I \delta(x)=[\min (\tilde{\delta}(\underline{x}), \tilde{\delta}(\bar{x})), \tilde{\delta}(\bar{x})]$ is the range norm of $x$, after only considering the finite component.

Problem 2.2. Given that matrix $A \in I(\mathbb{R})_{\varepsilon}^{m \times n}$, solve:

$$
\begin{aligned}
& \text { minimize } I \delta(b) \\
& \text { subject to } b \in \operatorname{Im}(A) \text {. }
\end{aligned}
$$

Definition 2.5. Given that matrix $b \in I(\mathbb{R})_{\varepsilon}^{m}$ with $b \approx[\underline{b}, \bar{b}]$. The function $T \delta(b)$ is said minimum if and only if $\tilde{\delta}(\underline{b})$ and $\tilde{\delta}(\bar{b})$ are minimum. 


\section{Minimizing the range norm if its image vector is finite}

The first we investigate minimizing the range norm if its image vector is finite, i.e., Problem 2.1. The next lemmas, definition and theorems are needed to answer Problem 2.1:

Lemma 2.6. Suppose that $A \in I(\mathbb{R})_{\mathcal{\varepsilon}}^{m \times n}$ with $A \approx[\underline{A}, \bar{A}]$. If $A=\left[A_{i j}\right]$ with $A_{i j} \neq[\varepsilon, \varepsilon]$ for each $i \in M, j \in N ; b \approx[\underline{b}, \bar{b}]$ and $0 \approx[\underline{0}, \overline{0}]$ with $\underline{b}=\underline{A} \otimes\left(\underline{A}^{*} \otimes^{\prime} \underline{0}\right)$ and $\bar{b}=\bar{A} \otimes\left(\bar{A}^{*} \otimes \prime \overline{0}\right)$, then

(i) $b \leq 0$ and

(ii) $b_{i}=0$ for some $i \in M$.

Proof. Suppose that $b \approx[\underline{b}, \bar{b}]$ with $\underline{b}=\underline{A} \otimes\left(\underline{A}^{*} \otimes{ }^{\prime} \underline{0}\right)$ and $\bar{b}=\bar{A} \otimes$ $\left(\bar{A}^{*} \otimes^{\prime} \overline{0}\right)$. Since $A=\left[A_{i j}\right]$ with $A_{i j} \neq[\varepsilon, \varepsilon]$ for each $i \in M$ and $j \in N$, $\underline{A}=\left[\underline{A}_{i j}\right]$ with $\underline{A}_{i j} \neq \varepsilon$ for each $i \in M$ and $j \in N$ and $\bar{A}=\left[\bar{A}_{i j}\right]$ with $\bar{A}_{i j} \neq \varepsilon$ for each $i \in M$ and $j \in N$. According to concept in max-plus algebra,

(i) $\underline{b} \leq \underline{0}$ and $\bar{b} \leq \overline{0}$,

(ii) $\underline{b}_{i}=\underline{0}$ and $\overline{b_{i}}=\overline{0}$ for some $i \in M$.

Since $b \approx[\underline{b}, \bar{b}]$ and $0 \approx[0, \overline{0}]$,

(i) $b \leq 0$,

(ii) $b_{i}=0$ for some $i \in M$.

Lemma 2.7. If $x, y \in I(\mathbb{R})^{m}$ and $a \in I(\mathbb{R})$ with $x \approx[\underline{x}, \bar{x}], y \approx[\underline{y}, \bar{y}]$ and $a \approx[\underline{a}, \bar{a}]$, then

(i) $I \delta(x \bar{\oplus} y) \leq I \delta(x) \bar{\oplus} I \delta(y)$,

(ii) $I \delta(x)=I \delta(a \bar{\otimes} x)$. 
Proof. Since $x \approx[\underline{x}, \bar{x}], y \approx[\underline{y}, \bar{y}]$ and $a \approx[\underline{a}, \bar{a}], x \bar{\oplus} y \approx[\underline{x} \oplus \underline{y}, \bar{x} \oplus \bar{y}]$ and $a \bar{\otimes} x \approx[\underline{a} \otimes \underline{x}, \bar{a} \otimes \bar{x}]$. Next, according to concept in max-plus algebra,

(i) $\delta(\underline{x} \oplus \underline{y}) \leq \delta(\underline{x}) \oplus \delta(\underline{y})$ and $\delta(\bar{x} \oplus \bar{y}) \leq \delta(\bar{x}) \oplus \delta(\bar{y})$,

(ii) $\delta(\underline{x})=\delta(\underline{a} \otimes \underline{x})$ and $\delta(\bar{x})=\delta(\bar{a} \otimes \bar{x})$.

Therefore,

(i)

$$
\begin{aligned}
I \delta(x \bar{\oplus} y) & =[\min (\delta(\underline{x} \oplus \underline{y}), \delta(\bar{x} \oplus \bar{y})), \delta(\bar{x} \oplus \bar{y})] \\
& \leq[\min (\delta(\underline{x}) \oplus \delta(\underline{y}), \delta(\bar{x}) \oplus \delta(\bar{y})), \delta(\bar{x}) \oplus \delta(\bar{y})] \\
& =[\min (\delta(\underline{x}), \delta(\bar{x})), \delta(\bar{x})] \oplus[\min (\delta(\underline{y}), \delta(\bar{y})), \delta(\bar{y})] \\
& =I \delta(x) \bar{\oplus} I \delta(y),
\end{aligned}
$$

(ii)

$$
\begin{aligned}
I \delta(x) & =[\min (\delta(\underline{x}), \delta(\bar{x})), \delta(\bar{x})] \\
& =[\min (\delta(\underline{a} \otimes \underline{x}), \delta(\bar{a} \otimes \bar{x})), \delta(\bar{a} \otimes \bar{x})]=I \delta(a \bar{\otimes} x) .
\end{aligned}
$$

Definition 2.8. The interval matrix $A \in I(\overline{\mathbb{R}})^{m \times n}, \quad A \approx[\underline{A}, \bar{A}]$ is considered as double $I(\mathbb{R})$-astic if $A$ is double $\mathbb{R}$-astic for each $A \in[\underline{A}, \bar{A}]$.

Theorem 2.9. The matrix $A \in I(\overline{\mathbb{R}})^{m \times n}$ with $A \approx[\underline{A}, \bar{A}]$ is double $I(\mathbb{R})$-astic if and only if $\underline{A}$ is double $\mathbb{R}$-astic.

Proof. It is known that $A \in I(\overline{\mathbb{R}})^{m \times n}$ with $A \approx[\underline{A}, \bar{A}]$ is double $I(\mathbb{R})$-astic. Based on the definition, $A$ is double $\mathbb{R}$-astic for each $A \in[\underline{A}, \bar{A}]$. If $A=\underline{A}$, then $\underline{A}$ is double $\mathbb{R}$-astic. It is known that $\underline{A}$ is double $\mathbb{R}$-astic. Since $\underline{A}$ is the lower bound matrix for the matrix interval $[\underline{A}, \bar{A}], \underline{A} \leq \bar{A}$. Therefore, since it is a matrix $\underline{A}$ double $\mathbb{R}$-astic, matrix $A$ 
double $\mathbb{R}$-astic for each $A \in[\underline{A}, \bar{A}]$, thus $A \in I(\overline{\mathbb{R}})^{m \times n}$ double $I(\mathbb{R})$-astic is obtained.

The following theorem presents solution to Problem 2.1:

Theorem 2.10. Suppose that $A \in I(\mathbb{R})_{\varepsilon}^{m \times n}$ is double $I(\mathbb{R})$-astic and $v_{a} \in I(\mathbb{R})^{m}$ is a vector whose each component is equal to $a \in I(\mathbb{R})$. Then $b \approx[\underline{b}, \bar{b}]$ is a solution for Problem 2.1 such that $\underline{b} \leq \underline{A} \otimes\left(\underline{A}^{*} \otimes^{\prime} \underline{v}_{\underline{a}}\right)$ and $\bar{b}=\bar{A} \otimes\left(\bar{A}^{*} \otimes \bar{v}_{\bar{a}}\right)$.

Proof. Suppose that $A \approx[\underline{A}, \bar{A}], v_{a} \approx\left[\underline{v}_{\underline{a}}, \bar{v}_{\bar{a}}\right]$ and $a \approx[\underline{a}, \bar{a}]$. Since $A \in I(\mathbb{R})_{\mathcal{\varepsilon}}^{m \times n}$ is double $I(\mathbb{R})$-astic, $v_{a} \in I(\mathbb{R})^{m}$ and $v_{a}$ is a vector whose each component is equal to $a \in I(\mathbb{R})$, which means that:

(i) Matrix $\underline{A} \in \mathbb{R}_{\varepsilon}^{m \times n}$ is double $\mathbb{R}$-astic and $\underline{v}_{a}$ is a vector whose component is equal to $\underline{a} \in \mathbb{R}$. Based on concept in max-plus algebra, $\underline{A} \otimes$ $\left(\underline{A}^{*} \otimes^{\prime} \underline{v}_{\underline{a}}\right)$ is a solution for Problem 1.1.

(ii) Matrix $\bar{A} \in \mathbb{R}_{\varepsilon}^{m \times n}$ is double $\mathbb{R}$-astic and $\bar{v}_{\bar{a}}$ is a vector whose component is equal to $\bar{a} \in \mathbb{R}$. Based on concept in max-plus algebra, $\bar{A} \otimes$ $\left(\bar{A}^{*} \otimes^{\prime} \bar{v}_{\bar{a}}\right)$ is a solution for Problem 1.1.

Therefore, $[\underline{b}, \bar{b}]$ with $\underline{b} \leq \underline{A} \otimes\left(\underline{A}^{*} \otimes^{\prime} \underline{v}_{\underline{a}}\right)$ and $\bar{b}=\bar{A} \otimes\left(\bar{A}^{*} \otimes \bar{v}_{\bar{a}}\right)$ is a solution for Problem 2.1.

\section{Minimizing the range norm if its image vector is not finite}

To solve Problem 2.2, the following theorem is used:

Theorem 2.11. Suppose that $A \in I(\mathbb{R})_{\varepsilon}^{m \times n}$, where $A=\left[A_{i j}\right]$ for each $i \in M$ and $j \in N$ with $A_{i j} \neq[\varepsilon, \varepsilon]$, and $v_{a} \in I(\mathbb{R})^{m}$ is a vector whose each component is equal to constant interval a. If $x \in I(\mathbb{R})_{\varepsilon}^{n}$ such that $b^{\prime} \leq$ 
$A \bar{\otimes} x$ is a solution for Problem 2.2, then $\forall j \in N$ satisfies:

a. $x_{j}=[\varepsilon, \varepsilon]$ or

b. $x_{j}=\left[\min \left(\left(\underline{A}^{*} \otimes^{\prime} \underline{v}_{\underline{a}}\right)_{j},\left(\bar{A}^{*} \otimes{ }^{\prime} \bar{v}_{\bar{a}}\right)_{j}\right),\left(\bar{A}^{*} \otimes^{\prime} \bar{v}_{\bar{a}}\right)_{j}\right]_{j}$.

Proof. Suppose that $A \approx[\underline{A}, \bar{A}], v_{a} \approx\left[\underline{v}_{\underline{a}}, \bar{v}_{\bar{a}}\right]$ and $a \approx[a, \bar{a}]$, Since $A=\left[A_{i j}\right]$ for each $i \in M$ and $j \in N$ with $A_{i j} \neq[\varepsilon, \varepsilon]$, and $v_{a} \in I(\mathbb{R})^{m}$ is a vector whose each component is equal to constant interval $a$, it means that:

(i) Matrix $\underline{A}=\left[\underline{A}_{i j}\right]$ for each $i \in M$ and $j \in N$ with $\underline{A}_{i j} \neq \varepsilon$ and $\underline{v}_{\underline{a}} \in \mathbb{R}^{m}$ is a vector whose each component is equal to constant $\underline{a}$.

(ii) Matrix $\bar{A}=\left[\bar{A}_{i j}\right]$ for each $i \in M$ and $j \in N$ with $\bar{A}_{i j} \neq \varepsilon$ and $\bar{v}_{\bar{a}} \in \mathbb{R}^{m}$ is a vector whose each component is equal to constant $\bar{a}$.

Then, suppose that $x \approx[\underline{x}, \bar{x}], b^{\prime} \approx\left[\underline{b}^{\prime}, \bar{b}^{\prime}\right]$ and $x_{j} \approx\left[\underline{x}_{j}, \bar{x}_{j}\right]$. It is known that $x \in I(\mathbb{R})_{\varepsilon}^{n}$ such that $b^{\prime}=A \bar{\otimes} x$ is a solution for Problem 2.2, which according to concept in max-plus algebra means:

(iii) $\underline{x} \in \mathbb{R}_{\varepsilon}^{n}$ with $\underline{b}^{\prime} \leq \underline{A} \otimes \underline{x}$ is the solution for Problem 1.2 , so $\forall j \in N$ satisfies:

$\mathrm{a}^{\prime} . \underline{x}_{j}=\varepsilon$ or

$\mathrm{b}^{\prime} . \underline{x}_{j}=\min \left(\left(\underline{A}^{*} \otimes^{\prime} \underline{v}_{\underline{a}}\right)_{j},\left(\bar{A}^{*} \otimes \bar{v}_{\bar{a}}\right)_{j}\right)$.

(iv) $\bar{x} \in \mathbb{R}_{\varepsilon}^{n}$ with $\bar{b}^{\prime}=\bar{A} \otimes \bar{x}$ is the solution for Problem 1.2 , so $\forall j \in N$ satisfies:

$\mathrm{a}^{\prime} . \bar{x}_{j}=\varepsilon$ or

$\mathrm{b}^{\prime} . \bar{x}_{j}=\left(\bar{A}^{*} \otimes \bar{v}_{\bar{a}}\right)_{j}$. 
Thus, from (i) and (iii) and also from (ii) and (iv), we have:

If $x \in I(\mathbb{R})_{\varepsilon}^{n}$ such that $b^{\prime}=A \bar{\otimes} x$ is the solution for Problem 2.2, then $\forall j \in N$ satisfies:
a. $x_{j}=[\varepsilon, \varepsilon]$ or
b. $x_{j}=\left[\min \left(\left(\underline{A}^{*} \otimes^{\prime} \underline{v}_{\underline{a}}\right)_{j},\left(\bar{A}^{*} \otimes{ }^{\prime} \bar{v}_{\bar{a}}\right)_{j}\right),\left(\bar{A}^{*} \otimes^{\prime} \bar{v}_{\bar{a}}\right)_{j}\right]_{j}$.

B. Maximizing the range norm of matrix image set over interval maxplus algebra

The problem of maximizing the range norm of matrix image set can be formulated as follows:

Problem 2.3. Given that matrix $A \in I(\mathbb{R})_{\varepsilon}^{m \times n}$, solve:

$$
\begin{aligned}
& \text { maximize } I \delta(b) \\
& \text { subject to } b \in \operatorname{Im}(A) \text {. }
\end{aligned}
$$

Definition 2.12. Given that matrix $b \in I(\mathbb{R})_{\varepsilon}^{m}$ with $b \approx[\underline{b}, \bar{b}]$. The function $I \delta(b)$ is said to be maximum if and only if $\delta(\bar{b})$ and $\delta(\underline{b})$ are maximum.

\section{Maximizing the range norm if its matrix is finite}

In this section, we discuss maximizing the range norm if its matrix is finite.

Theorem 2.13. Given that matrix $A \in I(\mathbb{R})^{m \times n}$ with $A=\left(A_{1} A_{2} \cdots A_{n}\right)$ and $A_{j} \approx\left[\underline{A}_{j}, \bar{A}_{j}\right] ; j=1,2, \ldots, n$; then for each $b \in \operatorname{Im}(A)$, $I \delta(b) \leq\left[\min \left(\max _{j=1,2, \ldots, n} \delta\left(\underline{A}_{j}\right), \max _{j=1,2, \ldots, n} \delta\left(\bar{A}_{j}\right)\right), \max _{j=1,2, \ldots, n} \delta\left(\bar{A}_{j}\right)\right]$.

Proof. Suppose that $A \in I(\mathbb{R})^{m \times n}$ and $b \in \operatorname{Im}(A)$ with $A \approx[\underline{A}, \bar{A}]$ and $b \approx[\underline{b}, \bar{b}]$, it means that $\underline{A}, \bar{A} \in \mathbb{R}^{m \times n}$ and $\underline{b} \in \operatorname{Im}(\underline{A}), \bar{b} \in \operatorname{Im}(\bar{A})$. Based 
on concept in max-plus algebra, it is obtained that:

$$
\begin{aligned}
\delta(\underline{b}) & \leq \max _{j=1,2, \ldots, n} \delta\left(\underline{A}_{j}\right) \\
& =\max _{j=1,2, \ldots, n}\left(\max _{i=1,2, \ldots, m} \underline{a}_{i j}-\min _{i=1,2, \ldots, m} \underline{a}_{i j}\right) \text { and } \\
\delta(\bar{b}) & \leq \max _{j=1,2, \ldots, n} \delta\left(\bar{A}_{j}\right) \\
& =\max _{j=1,2, \ldots, n}\left(\max _{i=1,2, \ldots, m} \bar{a}_{i j}-\min _{i=1,2, \ldots, m} \bar{a}_{i j}\right) .
\end{aligned}
$$

Therefore,

$$
\begin{aligned}
I \delta(b)= & {[\min (\delta(\underline{b}), \delta(\bar{b})), \delta(\bar{b})] } \\
\leq & {\left[\min \left(\max _{j=1,2, \ldots, n} \delta\left(\underline{A}_{j}\right), \max _{j=1,2, \ldots, n} \delta\left(\bar{A}_{j}\right)\right), \max _{j=1,2, \ldots, n} \delta\left(\bar{A}_{j}\right)\right] } \\
= & {\left[\operatorname { m i n } \left(\max _{j=1,2, \ldots, n}\left(\max _{i=1,2, \ldots, m} \underline{a}_{i j}-\min _{i=1,2, \ldots, m} \underline{a}_{i j}\right),\right.\right.} \\
& \left.\max _{j=1,2, \ldots, n}\left(\max _{i=1,2, \ldots, m} \bar{a}_{i j}-\min _{i=1,2, \ldots, m} \bar{a}_{i j}\right)\right), \\
& \left.\max _{j=1,2, \ldots, n}\left(\max _{i=1,2, \ldots, m} \bar{a}_{i j}-\min _{i=1,2, \ldots, m} \bar{a}_{i j}\right)\right] .
\end{aligned}
$$

\section{Maximizing the range norm if its matrix is not finite}

In the last section, we discuss maximizing the range norm if its matrix is not finite.

Theorem 2.14. Given that $A \in I(\mathbb{R})_{\mathcal{\varepsilon}}^{m \times n}$ is double $I(\mathbb{R})$-astic and not finite, i.e., $\exists i \in M, j \in N$ such that $a_{i j}=[\varepsilon, \varepsilon]$, then Problem 2.3 is unbounded.

Proof. Suppose that $A \in I(\mathbb{R})_{\varepsilon}^{m \times n}$ with $A \approx[\underline{A}, \bar{A}]$ is double $I(\mathbb{R})$-astic and infinite so $\exists i \in M, j \in N$ such that $a_{i j}=[\varepsilon, \varepsilon]$ which means that $\underline{A}, \bar{A} \in \mathbb{R}_{\varepsilon}^{m \times n}$ is double $\mathbb{R}$-astic and $\exists i \in M, j \in N$ such that $\underline{a}_{i j}=\varepsilon$ and 
$\bar{a}_{i j}=\varepsilon$. Therefore, according to concept in max-plus algebra, Problem 1.3 is related to matrices $\underline{A}$ and $\bar{A}$ which have unbounded solution, namely:

a. Given that matrix $\underline{A} \in \mathbb{R}_{\varepsilon}^{m \times n}$, solve:

maximize $\delta(\underline{b})$

subject to $\underline{b} \in \operatorname{Im}(\underline{A})$.

b. Given that matrix $\bar{A} \in \mathbb{R}_{\varepsilon}^{m \times n}$, solve:

maximize $\delta(\bar{b})$

subject to $\bar{b} \in \operatorname{Im}(\bar{A})$.

Therefore, Problem 2.3, that is given matrix $A \in I(\mathbb{R})_{\varepsilon}^{m \times n}$, solve:

maximize $I \delta(b)$

subject to $b \in \operatorname{Im}(A)$

has unbounded solution.

\section{Concluding Remarks}

The main results are obtained the way to:

a. Minimizing the range norm of matrix image set over interval max-plus algebra, with its finite and not finite image vector.

These results are presented in Theorem 2.10 and Theorem 2.11.

b. Maximizing the range norm of matrix image set over max-plus algebra, with its finite and not finite matrix.

These results are presented in Theorem 2.13 and Theorem 2.14. 


\section{References}

[1] M. Akian, G. Cohen, S. Gaubert, J. P. Quadrat and M. Viot, Max-plus algebra and applications to system theory and optimal control, Proceedings of the International Congress of Mathematicians, Zurich, Switzerland, 1994.

[2] M. H. Andersen, Max-plus algebra: properties and applications, A Thesis Submitted to the Department of Mathematics in Partial Fulfillment of the Requirements for the Degree of Master of Science in Mathematics, 2002.

[3] F. Bacelli, G. Cohen, J. G. Older and J. P. Quadat, Synchronization and Linearity, John Wiley \& Sons, New York, 2001.

[4] P. Butkovic and K. P. Tam, On some properties of the image of a max linear mapping, Contemp. Math. 495 (2009), 115-126.

[5] R. A. Cuninghame-Green and P. Butkovic, Bases in max-algebra, Linear Algebra Appl. 389 (2004), 107-120.

[6] K. G. Farlow, Max-plus algebra, Master's Thesis Submitted to the Faculty of the Virginia Polytechnic Institute and State University in Partial Fulfillment of the Requirements for the Degree of Masters in Mathematics, 2009,

[7] Z. R. Konigsberg, A generalized eigenmode algorithm for reducible regular matrices over the max-plus algebra, Int. Math. Forum 4(24) (2009), 1157-1171.

[8] V. Kreinovich, Why intervals? Why fuzzy numbers? Towards a new justification, Int. J. Inform. Technol. Intell. Comput. 2(1) (2007), 113-119.

[9] M. A. Rudhito, Fuzzy number max-plus algebra and its application to fuzzy scheduling and queuing network problems, The Study Program S3 Mathematics Faculty of Mathematics and Natural Sciences, Gadjah Mada University, 2011 (Indonesian).

[10] S. Sergeev, Max-plus definite matrix closures and their eigenspaces, Linear Algebra Appl. 421(2-3) (2007), 182-201.

[11] Siswanto, A. Suparwanto and M. A. Rudhito, Minimizing range norm of the image set of matrix over interval max-plus algebra, Proceedings of the National Seminar on Mathematics and Applications, Department of Mathematics, Faculty of Science and Technology, Airlangga University, 2013 (Indonesian).

[12] K. P. Tam, Optimizing and approximating eigenvectors in max algebra thesis, A Thesis Submitted to The University of Birmingham for the Degree of Doctor of Philosophy (Ph.D.), 2010. 